\title{
Ley 6/2013, de Cooperativas de Cantabria: contenido y oportunidad para el desarrollo de la economía social *
}

\author{
Dr. Enrique Gadea \\ Universidad de Deusto
}

Sumario: I. Introducción. II. Sobre los intereses convergentes y el modelo de sociedad cooperativa. III. Directrices más relevantes de la Ley de Cooperativas de Cantabria: 1. Constitución de la cooperativa. 2. Número de socios. 3. Régimen orgánico. 4. El capital social. 5. La regulación de la imputación de pérdidas. 6. El Fondo de Reserva Obligatorio (FRO). 7. Clases de cooperativas. 8. El Consejo Cántabro de la Economía. IV. Reflexión final.

Resumen: Con la LCCANT se pretende ofrecer una regulación respetuosa con el modelo de verdadera sociedad cooperativa y, al mismo tiempo, introducir los instrumentos necesarios, no sólo para que las cooperativas puedan superar una etapa de crisis sino para que la organización cooperativista pueda ocupar un papel relevante dentro de la actividad económica cántabra. Sin embargo, aprobar una legislación adecuada es sólo el primer paso; una vez aprobada la norma empieza el verdadero reto. Entonces, el Gobierno regional deberá dotarse de una estructura administrativa capaz de ofrecer servicios y soluciones al mundo cooperativo y apostar, de forma decidida, por la divulgación del ideario cooperativo y por la promoción de las sociedades cooperativas como fórmula ventajosa respecto a otras formas jurídicas, favorecedora del autoempleo.

Palabras clave: Directrices y Ley de Cooperativas de Cantabria.

Abstract: The LCCANT (Cantabria Law on Cooperatives) intends to provide regulation that respects the model of the true cooperative company while introducing the instruments that cooperatives need, not only to overcome a period of crisis, but to play a relevant role in Cantabria's economy. However, passing adequate legislation is only the first step. The true challenge begins afterwards. The regional government should equip itself with an administrative

* Texto basado en la ponencia presentada en el XV Congreso de Investigadores en Economía Social de CIRIEC-España, celebrado en Santander en septiembre de 2014. 
structure that can offer cooperatives services and solutions and actively engage in disseminating their ideals. Furthermore, it should promote cooperative companies as an advantageous arrangement in comparison to other types of business organisation.

Key words: Guidelines and the Cantabria Law on Cooperatives. 


\section{Introducción}

Superados los prejuicios y las resistencias de aquellos que defienden un concepto de empresa anclado en una visión narcisista donde la única meta es la maximización del lucro, se presenta como alternativa la sociedad cooperativa que, con una adecuada política de fomento, puede constituir un instrumento importante para mejorar el empleo y el nivel de bienestar de los ciudadanos.

En España, las cooperativas emplean a más de 270.000 personas y su actividad, sumada a la de las demás entidades que conforman la economía social, supone más del $10 \%$ del PIB. La tasa de paro en las cooperativas está seis puntos por debajo del nivel general. El empleo en las cooperativas es más estable gracias a la flexibilidad laboral y a la moderación salarial, y por ello, en este ámbito, se han producido menos despidos durante la crisis. Los empresarios de la economía social mantienen una tasa de empleo fijo de un $80 \%$, lo que convierte a las empresas de este sector en un modelo empresarial único que se preocupa tanto de las condiciones laborales de la empresa como de las del trabajador.

Siendo ello así y en el contexto actual de necesidad de búsqueda de autoempleo a través de este cauce, el Gobierno de Cantabria, en consonancia, además, con la demanda de las distintas Asociaciones del sector, debía apostar de forma decidida por el modelo cooperativo aprobando una norma propia. Para ello, en septiembre de 2012, la Dirección General de Trabajo del Gobierno de Cantabria, dependiente de la Consejería de Economía, Hacienda y Empleo, decide crear una Comisión de Trabajo para preparar un Borrador de Anteproyecto de Ley de Cooperativas de Cantabria.

\section{Sobre los intereses convergentes y el modelo de sociedad cooperativa}

A la hora de elaborar el Borrador, los miembros de la Comisión redactora del texto se encuentran con la necesidad de conciliar dos intereses principales, que no tienen por qué ser necesariamente divergentes. Por una parte, el del sector cooperativo, que, como es lógico, quiere ver satisfechas sus necesidades y reflejadas sus visiones en su texto legal propio. Y, por otra, el interés público, que requiere velar por la construcción de una institución rigurosa en sus planteamientos sociales y, a la vez, tecnificada, permeable a la especialización y sensible al mercado. 
La Comisión desarrolla su trabajo sobre la base de que el elemento definitorio de la cooperativa es que constituye una iniciativa empresarial por parte de un grupo de personas que, al margen de la participación capitalista de cada socio, tiene por finalidad la satisfacción de sus necesidades mediante la recuperación de la forma de empresa personalista y de funcionamiento interno democrático. Ese es el hecho diferencial sobre el que debe basarse el adecuado tratamiento (incluso en el plano fiscal) de la sociedad cooperativa. La especial estructura participativa de las cooperativas se vértebra en torno a tres pilares:

1. El papel del socio. En la cooperativa, los socios participan no sólo aportando las sumas de capital necesarias sino operando como clientes, en las cooperativas de consumo, y como trabajadores, en las cooperativas de producción.

2. La gestión democrática por parte de sus miembros, basada en la asociación igualitaria, que, en la práctica, se concreta, al menos en las cooperativas de primer grado, en la regla general de que los socios tengan iguales derechos de voto (un socio, un voto).

3. El sistema de distribución de beneficios al margen de la contribución en el capital, en proporción a las operaciones, servicios o actividades realizadas por cada socio en la entidad.

Desde el punto de vista de una adecuada técnica legislativa, como es sabido, coincidimos con nuestra doctrina mayoritaria (ARROYO, BORJABAD, DIVAR, VICENT CHULIÁ, PANIAGUA, etc.) en que la fragmentación de nuestra Legislación cooperativa es perjudicial para una sociedad que tiene que competir con instituciones capitalistas que tienen una regulación uniforme y difícilmente explicable ante el mandato de fomentar las sociedades cooperativas que impone el artículo 129.2 de la CE. E, igualmente, en que la existencia de tantas leyes de cooperativas en España va en contra de la propia lógica jurídica. Es comúnmente aceptado que las CCAA, en materia de cooperativas, no necesitan regular ex novo y totalmente todo lo relativo a las mismas. Ciertamente, no se ve ninguna necesidad de que materias como el concepto legal de sociedad cooperativa, el régimen de los órganos de gestión y control de la cooperativa y de verificación o control de cuentas, el régimen del capital, las causas de disolución o el procedimiento de liquidación sean distintas en cada uno de los territorios autónomos. Es por ello por lo que no nos hemos visto obligados a realizar ningún alarde legislativo (a introducir aspectos necesariamente distintos a otros leyes) sino más bien a recoger las soluciones que hemos considerado más adecuadas para un tipo societario (en definitiva, un operador eco- 
nómico) que debe competir en el mercado con las sociedades capitalistas, bajo la óptica de unos valores y unos principios distintos.

\section{Directrices más relevante de la Ley de Cooperativas de Cantabria}

\section{Constitución de la cooperativa}

En el procedimiento de constitución de la sociedad cooperativa se permite que los promotores elijan entre dos vías: la celebración de una asamblea constituyente, o la constitución directa mediante otorgamiento de escritura pública. No obstante, sin perjuicio de lo anterior, la adquisición de su personalidad jurídica plena se supedita a la doble exigencia de escritura pública e inscripción en el Registro de Sociedades Cooperativas de Cantabria. En el momento constitutivo, de especial relevancia para la vida de la sociedad, la exigencia de escritura pública es importante. La escritura dará precisión jurídica al negocio constituido, tanto para la seguridad de los socios como de los terceros con los que tiene que relacionarse en su vida económica. Con la escritura pública se reúnen las ventajas de concreción de fines, autorregulación normativa de la persona jurídica, permanencia del negocio de constitución y trascendencia de su validez frente a terceros, autenticidad formal en el tráfico jurídico y conservación de la empresa.

\section{Número de socios}

En España lo habitual era exigir cinco socios como mínimo para constituir una cooperativa, cifra que fue reducida a tres por la LCOOP (artículo 8), que es el criterio seguido por la mayoría de leyes autonómicas; y también por la LCCANT, que prevé que las sociedades cooperativas de primer grado estarán integradas como mínimo por tres socios (artículo 5). Sin embargo, la particularidad de la LCCANT con respecto a la LCOOP es que se señala expresamente que los tres socios deben realizar la actividad cooperativizada; es decir, debe tratarse de tres socios ordinarios, no computándose, por tanto, a estos efectos los socios temporales, colaboradores e inactivos.

La notable reducción en el número mínimo de socios legalmente exigido para constituir una cooperativa de primer grado (recordemos que la Ley de cooperativas de 1931 exigía veinte, la de 1942 quince, 
la de 1974 siete y la LGC de 1987 cinco) se debe interpretar como una medida de fomento de estas sociedades, para permitir así poder abordar bajo esta forma societaria proyectos que requieren para su viabilidad un número muy limitado de personas. Este proceso que sigue su curso, como lo demuestra la admisión de sólo dos socios para constituir las cooperativas especiales de Extremadura (Ley 8/2006) o las pequeñas del País Vasco (Ley 6/2008), también ha sido tomado en consideración por la LCCANT. En efecto, en el contexto actual favorable a las ayudas al autoempleo, la LCCANT ha apostado decididamente por el modelo cooperativo flexibilizando todavía más la constitución de estas sociedades, al permitir la creación de cooperativas de trabajo de dos socios (artículo 100).

\section{Régimen orgánico}

La LCCANT, al igual que la LCOOP de 1999, dedica un artículo -el 30- a enumerar los órganos de la sociedad cooperativa. Según prescribe el referido artículo 30, la estructura orgánica del tipo social se construye en torno a dos órganos necesarios, que son la asamblea general y el consejo rector. Asimismo señala que los estatutos podrán prever la existencia de órganos facultativos: un órgano de intervención, un comité de recursos y otras instancias de carácter consultivo, siempre que sus funciones no se confundan con las propias de los órganos sociales necesarios. De ese modo, apartándose de lo previsto en la LCOOP, la LCCANT permite la sustitución del órgano de intervención por un sistema de censura de cuentas ejercitado por profesionales independientes (auditoría externa de las cuentas anuales: artículo 77). Llegado este punto, los términos del debate se desplazan a si no debería prescindirse de la obligatoriedad de la auditoría externa por el mero hecho de no contemplar en los estatutos la existencia del órgano de intervención, entre otros aspectos, para no gravar a las sociedades cooperativas frente a otras de parecida tipología, como las sociedades laborales o las SRL. La reivindicación del sector en este punto parece razonable, por lo que debería valorarse nuevamente la eliminación de esa obligación en defecto de órgano de intervención en una futura reforma del texto legal.

Con la finalidad de instaurar un sistema flexible para las pequeñas cooperativas, la LCCANT permite, en aquellas cuyo número de socios no sea superior a diez, que la representación, gobierno y gestión de la cooperativa se confíe a un administrador único o a dos administradores, que actúen mancomunada o solidariamente (artículo 43.2). 
Al igual que en la derogada LSRL de 1995 (y el actual artículo 161 de la LSC) y a diferencia de la Ley vasca de cooperativas de 1993 que establece un reparto competencial con carácter imperativo (artículos 31.3 y 4 y 40.1), la LCCANT, como la LCCOP, sigue el modelo flexible de la sociedad de responsabilidad limitada alemana, que reconoce expresamente a los socios una amplia posibilidad de influir en la administración. El legislador ha considerado que la sociedad cooperativa regulada por la LCCANT debe ser un tipo social en el que el marco estatutario debe permitir una mayor capacidad de modulación de la presencia de los socios en las decisiones sociales en función de las particulares necesidades que cada sociedad pretende atender o encauzar.

De lo establecido en el artículo 32.1 y 2 (ap. k) se deduce que los estatutos pueden configurar distintos modelos de relación de competencia en materia de gestión, y así:

1. Se puede fijar un modelo plenamente corporativo, que potencie y garantice la autonomía de los administradores, eliminando expresamente el poder de instrucción de la asamblea general y las reservas de autorización de decisiones o acuerdos sobre determinados asuntos de gestión en favor del citado órgano.

2. Se puede fijar un modelo personalista e intervencionista en favor de los socios (órgano administrativo de carácter más propiamente ejecutivo), incluyendo amplias reservas estatutarias de autorización de medidas de gestión en favor de la asamblea general (medidas limitativas y de control) y utilizando el poder de instrucción (medidas impulsivas).

3. O se pueden fijar modelos intermedios basados en un equilibrio adaptado a las circunstancias de la empresa.

En relación con esta cuestión, el problema es determinar el margen de autonomía de los estatutos para ampliar la competencia de la asamblea general, limitando las facultades de los administradores en relación con asuntos de gestión. Aunque partiendo de posiciones muy favorables a la plena autonomía estatutaria y a la supremacía de la asamblea podría entenderse que, excluidas las facultades imperativamente atribuidas al órgano de administración (representación de la sociedad, formulación de las cuentas anuales, convocatoria de la asamblea, etc.), todas las demás facultades de gestión de la empresa podrían otorgarse por vía estatutaria a la asamblea general, de forma que los administradores se convirtieran en meros ejecutores de los actos decididos por los socios, nos parece que, al igual que en las SRL, no puede irse tan lejos. Coincidimos con G. Esteban Velasco en que «más bien hay que interpretar que la competencia de la junta (en este caso 
de la asamblea) en materia de gestión no puede ampliarse hasta llegar a vaciar absolutamente de contenido la posición y la función legal de los administradores. En otros términos, los poderes de intervención sólo podrán referirse a asuntos concretos de especial importancia: actos o categorías de actos que se refieran a la estructura financiera de la empresa o que pertenezcan a la llamada administración extraordinaria. Incluso se puede pensar en ocasionales instrucciones sobre asuntos ordinarios, pero nunca injerencias que por su frecuencia o intensidad anulen la posición de los administradores con un mínimo de competencia gestora autónoma» ${ }^{1}$. La justificación de esa atribución se encuentra en la necesidad de que exista una instancia que pueda ser identificada como garante y responsable de una correcta y ordenada gestión.

La LCCANT, al igual que la LCOOP, admite que los socios puedan participar en la gestión de la empresa, mientras la Ley vasca atribuye la gestión de la cooperativa en exclusiva a los administradores (artículo 40.1). Este hecho pone de manifiesto las diferencias existentes en una y en otra norma. La estructura organizativa de las cooperativas reguladas por la Ley vasca está legalmente fijada y no puede ser alterada por la voluntad de los socios, a diferencia de la de las cooperativas reguladas por la LCCANT en que la organización está en gran medida a disposición de los socios, de forma que pueden por sí mismos determinar el destino de la sociedad y de la actividad empresarial desarrollada en forma societaria². Esas diferencias estructurales denotan una diferente configuración tipológica. En efecto, la normativa vasca, posiblemente influenciada por las cooperativas industriales existentes en su territorio y, en particular, por la Corporación de Mondragón, diseña un modelo de gestión más ágil, pensado para la empresa de cierta dimensión. En realidad, sigue las autorizadas voces que en nuestra doctrina ${ }^{3}$ han manifestado, en el ámbito de las sociedades anónimas, su postura crítica frente a la posibilidad de influencia de los socios en la administración, por considerar que la creciente complejidad y tecnificación del mundo empresarial exige una gestión profesionalizada, más ágil y efi-

1 «La estructura orgánica de la sociedad de responsabilidad limitada», Rds, 1994 (número extraordinario), pp. 399 y 400; y «La administración de la sociedad de responsabilidad limitada», en Tratado de la sociedad limitada, Fundación cultural del Notariado, Madrid, 1997, p. 713.

2 Sobre esta cuestión puede verse el artículo de OtXOA, R.: "La participación de los socios de la SRL en la gestión de la sociedad», RdS, 1994, 2, p. 102.

3 GarRigues/Uría, en Comentarios a la Ley de sociedades anónimas, II, Madrid, 1976, pp. 29 y 30, destacan las consecuencias negativas que en la práctica ha tenido la intervención de la junta en los asuntos de pura gestión «que deberían estar reservados a los administradores». 
caz que garantice la capacidad operativa de la empresa. Por el contrario, la LCCANT regula un modelo más flexible, dado que los estatutos pueden diseñar un modelo similar al descrito en la Ley vasca o bien otro, más personalista y teóricamente menos eficiente, pensado para empresas pequeñas o medianas, gestionadas por los propios socios, en ocasiones con la intervención de terceros, pero en el que los titulares de la empresa se reservan algún tipo de influencia sobre los administradores.

LCCANT, al igual que la LCOOP, admite que los socios puedan participar en la gestión de la empresa, mientras la Ley vasca atribuye la gestión de la cooperativa en exclusiva a los administradores (artículo 40.1). Este hecho pone de manifiesto las diferencias existentes en una y en otra norma. La estructura organizativa de las cooperativas reguladas por la Ley vasca está legalmente fijada y no puede ser alterada por la voluntad de los socios, a diferencia de la de las cooperativas reguladas por la LCCANT en que la organización está en gran medida a disposición de los socios, de forma que pueden por sí mismos determinar el destino de la sociedad y de la actividad empresarial desarrollada en forma societa$\mathrm{ria}^{4}$. Esas diferencias estructurales denotan una diferente configuración tipológica. En efecto, la normativa vasca, posiblemente influenciada por las cooperativas industriales existentes en su territorio y, en particular, por la Corporación de Mondragón, diseña un modelo de gestión más ágil, pensado para la empresa de cierta dimensión. En realidad, sigue las autorizadas voces que en nuestra doctrina ${ }^{5}$ han manifestado, en el ámbito de las sociedades anónimas, su postura crítica frente a la posibilidad de influencia de los socios en la administración, por considerar que la creciente complejidad y tecnificación del mundo empresarial exige una gestión profesionalizada, más ágil y eficaz que garantice la capacidad operativa de la empresa. Por el contrario, la LCCANT regula un modelo más flexible, dado que los estatutos pueden diseñar un modelo similar al descrito en la Ley vasca o bien otro, más personalista y teóricamente menos eficiente, pensado para empresas pequeñas o medianas, gestionadas por los propios socios, en ocasiones con la intervención de terceros, pero en el que los titulares de la empresa se reservan algún tipo de influencia sobre los administradores.

4 Sobre esta cuestión puede verse el artículo de OTXOA, R.: «La participación de los socios de la SRL en la gestión de la sociedad», RdS, 1994, 2, p. 102.

5 Garrigues/Uría, en Comentarios a la Ley de sociedades anónimas, II, Madrid, 1976, pp. 29 y 30, destacan las consecuencias negativas que en la práctica ha tenido la intervención de la junta en los asuntos de pura gestión «que deberían estar reservados a los administradores». 
En lo que sí existe coincidencia con la Ley vasca, al igual que con la LCOOP, en la ilimitabilidad del poder de representación de los administradores, ya que también establece que «en todo caso, las facultades representativas del consejo rector se extienden a todos los actos relacionados con la actividades que integren el objeto social de la cooperativa, sin que surjan efecto frente a terceros las limitaciones que en cuanto a ellos pudieran contener los estatutos» (artículo 43.4). En este punto, debe tenerse en cuenta que, al igual que en las sociedades anónimas, el ámbito de representación se extiende no sólo a los actos directamente comprendidos en el objeto social, sino también a aquellos otros (actos conexos) que constituyen medios auxiliares para el desarrollo de ese objeto (puede verse en ese sentido, las Res. de la D.G.R.N. de 16 de marzo de 1990, de 16 de marzo de 1992 y de 8 de julio de 1993). En la LCCANT se echa de menos la mención a lo qué sucede si los administradores realizan actos con terceros no comprendidos en el objeto social. En ese sentido, debería haberse incorporado la solución prevista en la Ley vasca que, mostrando una considerable preocupación por extender al máximo la protección del tercero de buena fe, establece la protección de éstos por actos «ultra vires» cuando señala —en el último inciso del artículo 40.2-: «la cooperativa quedará obligada frente a terceros que hayan obrado de buena fe y sin culpa grave, aunque se desprenda de los estatutos que el acto no está comprendido en el objeto social».

\section{El capital social}

La LCCANT exige un capital social mínimo para constituir una cooperativa: el artículo 6 señala que el capital social mínimo no será inferior a 3.000 euros. En el momento de la constitución, ese capital deberá hallarse totalmente suscrito, si bien se admite que el desembolso inicial se limite al veinticinco por ciento del total y que se difiera el resto durante el plazo que establezcan los estatutos o que decida la asamblea general, aunque, en cualquier caso, dentro del limite de cuatro años.

Con ello, la norma trata no sólo de alinearse con las recientes corrientes del Derecho societario comparado, sino también de subrayar el carácter empresarial de las cooperativistas y de ofrecer a los terceros, desde el nacimiento mismo de la entidad, un testimonio real de seriedad económica y de seguridad jurídica. Esta medida debe ser valorada muy positivamente, sobre todo en una sociedad de capital variable como la cooperativa, aunque más desde el punto de vista de la serie- 
dad exigible a la iniciativa económica que desde el punto de vista de la garantía que a los terceros puede ofrecer capital social, ya que es obvia la imposibilidad técnica de fijar a priori una cifra de capital que procure el soporte financiero preciso para toda iniciativa empresarial que pretenda organizarse a través de una sociedad cooperativa. La falta de vinculación entre esa cifra y los datos concretos de la empresa permite señalar que la exigencia de capital mínimo, al igual que en las sociedades capitalistas, no garantiza la fijación de una cifra de capital adecuada o, en términos más precisos, no evita el fenómeno de la infracapitalización de la sociedad motivado por la desproporción entre la magnitud del capital de responsabilidad fijado estatutariamente y el nivel de riesgo de la empresa que en cada caso se programe para llevar a efecto el objeto social.

\section{La regulación de la imputación de pérdidas}

La doctrina coincide en que la imputación de pérdidas constituye un sistema de saneamiento empresarial específico de las sociedades cooperativas, aunque discrepa sobre el alcance de la misma sobre el patrimonio de los socios:

1. Un sector considera que, si los estatutos no lo remedian, a los socios pueden imputárseles cualquier tipo de pérdidas sociales, por las que responden ilimitadamente. Para estos autores el principio de la responsabilidad limitada del socio solo tiene vigencia frente a terceros, y ello por el hecho de referirse expresamente el legislador a las deudas sociales, defendiendo que vía imputación de pérdidas los socios responden ilimitadamente frente a la Cooperativa, distinguiendo así entre responsabilidad interna y externa. Para ello, se parte de la consideración de que no es lo mismo hablar de deudas que de pérdidas.

2. En contra de esta posición, y a favor de la responsabilidad limitada, se señala como posible la cancelación de deudas por el abandono de capital a modo de pago por cesión de capital, pero no la exigencia directa de las perdidas al socio, por exigir consentimiento expreso e individual de cada socio, de conformidad con el artículo 15.3 de la LCOOP y concordantes, que prevén la responsabilidad limitada de éstos a las aportaciones al capital que hayan suscrito.

Como es sabido, en España no existe una única Ley de Cooperativas, si no que, junto a la Ley Estatal (LCOOP), se ha aprobado práctica- 
mente una Ley por Comunidad Autónoma, con orientaciones no siempre coincidentes.

En este sentido, destacar, en primer lugar, que las distintas Leyes contemplan dos sistemas de imputación en función de la procedencia de las pérdidas:

1. Las normas que regulan un sistema de imputación diferenciado para las pérdidas procedentes de la actividad económica realizada por la cooperativa con los socios y para resto de las pérdidas societarias (artículo 58.5 LCAR, artículo 69.4 LCCV artículo 63 LCEEx o artículo 61 LCCM).

2. Las normas que fijan un único sistema de compensación (artículo 59 LCOOP, artículo 69 LCPV y artículo 67.2 LCCAT).

En el segundo caso, el régimen legal de imputación se aplica a cualquier pérdida con independencia de su procedencia, por lo que todas ellas podrán ser imputadas, en último término, a los socios. Ello contrasta con lo previsto por las Leyes que aplican el sistema de imputación de pérdidas a las que derivan de la actividad cooperativizada con los socios, pero que no permiten imputar las demás (las derivadas de la actividad cooperativizada con terceros y las derivadas de actividades extraordinarias) a éstos directamente, sino que únicamente contemplan la compensación con cargo al fondo de reserva obligatorio (aunque esa es la regla general, cabe destacar que en el artículo 69.5 de la LCCV y en el 61 de la LCCM se prevé que las pérdidas extracooperativas y extraordinarias se imputarán a la reserva obligatoria y a las reservas voluntarias).

Según nuestro criterio, el sistema más acorde con una sociedad cooperativa no mutualista, que puede, por tanto, operar con socios o con terceros, con el objeto de extraer, si así lo desean sus socios, la máxima utilidad de sus estructuras organizativas, y caracterizada por un funcionamiento interno democrático y por un sistema de reparto de beneficios no capitalista, es el segundo. Bajo este modelo, consideramos que lo más procedente es que el particular sistema de compensación de pérdidas propio de la sociedad cooperativa se extienda a todas sus operaciones.

En segundo lugar, de las diferentes Leyes podemos extraer hasta tres orientaciones distintas sobre el alcance de la imputación de las pérdidas de la Cooperativa en el patrimonio de los socios:

1. Leyes que no se pronuncian sobre el alcance de la imputación. Dentro de este apartado cabe situar la Ley 27/1999, de 16 de julio, de Cooperativas (LCOOP) y la Ley 4/1993, de 24 de junio, 
de Cooperativas del País Vasco (LCPV). Estas normas prevén la responsabilidad limitada de socios a las aportaciones al capital que hayan suscrito (artículo 15.3 LCOOP y 56.1 LCPV), aunque, posteriormente, al regular la imputación de pérdidas (artículos 59 LCOOP y 69 LCPV) señalan que la cuantía no compensada con los fondos obligatorios y voluntarios se imputará a los socios en proporción a las operaciones, servicios o actividades realizadas por cada uno de ellos con la cooperativa. Las pérdidas imputadas a cada socio se satisfarán de alguna de las formas siguientes:

1. Directamente o mediante deducciones en sus aportaciones al capital social o, en su caso, en cualquier inversión financiera del socio en la cooperativa que permita esa imputación, dentro del ejercicio siguiente a aquél en que se hubiera producido.

2. Con cargo a los retornos que puedan corresponder al socio en los cinco años siguientes. Si quedasen pérdidas sin compensar éstas deberán satisfacerse por el socio en el plazo máximo de un mes.

Como puede verse, es cierto que la redacción de los preceptos es confusa y que, de lege lata y leyendo solo lo dispuesto en los artículos 59 LCOOP y 69 LCPV, puede mantenerse la imputación ilimitada de pérdidas al socio.

Las dudas son mayores si tomamos como referencia la Ley 2/1998, de 26 de marzo, de Sociedades Cooperativas de Extremadura (LCEEX), que en su artículo 63. 1b) prevé que:

«Las pérdidas imputadas a cada socio se satisfarán directamente, en el ejercicio económico siguiente a aquel en que se hayan producido, mediante deducciones en las aportaciones al capital social. También pueden satisfacerse con cargo a los retornos que podrían corresponder al socio en los cinco años siguientes; las pérdidas que, pasado dicho plazo, queden sin compensar serán satisfechas directamente por el socio, en el plazo de un mes».

$2 .^{\circ}$ Leyes que se decantan por la responsabilidad ilimitada del socio por las pérdidas de la Cooperativa derivadas de la actividad cooperativizada. En este apartado cabe incluir Ley 8/2003, de 24 de marzo, de Cooperativas de la Comunidad Valenciana (LCCV), en cuyo artículo 69.3 señala: "Si los estatutos sociales lo establecen, las pérdidas derivadas de la actividad cooperati- 
vizada con los socios que se imputen a éstos, alcanzarán como máximo el importe total de los anticipos asignados a los socios en el ejercicio económico, más sus aportaciones a capital social y su participación en las reservas repartibles». De lo que se deduce que, salvo previsión estatutaria, vía imputación de pérdidas los socios responden ilimitadamente frente a la Cooperativa, si bien únicamente, como ha quedado señalado, de las pérdidas derivadas de la actividad cooperativizada con los socios. Asimismo, y hasta con mayor claridad, el artículo 58.5.e) del Decreto Legislativo 2/2014, de 25 de agosto, por el que se aprueba el Texto Refundido de la Ley de Cooperativas de Aragón (LCAR) dispone que: "Las pérdidas asumidas por la Asamblea General y no compensadas serán consideradas como un crédito a favor de la cooperativa, que podrá ser ejercitado aunque el socio haya causado baja voluntaria u obligatoria en la misma».

3. Leyes que prevén expresamente que las pérdidas de la Cooperativa sólo podrán imputarse al socio hasta el límite de sus aportaciones al capital. La primera Ley que, de forma clara, garantiza la compatibilidad real de la responsabilidad limitada, que suelen prever las leyes de cooperativas modernas, y del sistema de imputación de pérdidas es la Ley 18/2002, de 5 de julio, de Cooperativas de Cataluña (LCCAT). Repárese en que, por una parte, en el artículo 54 dispone que: "Salvo que los estatutos sociales dispongan lo contrario, los socios han de responder de las deudas sociales de forma limitada a las aportaciones al capital social suscritas, tanto si son desembolsadas como si no»; y, por otra, en su artículo 67.4 prevé que: «Las pérdidas que, transcurrido el plazo al que se refiere el apartado 1, queden sin compensar deben satisfacerse directamente por el socio o socia en el plazo de un mes hasta el límite de sus aportaciones al capital, si no se insta a la quiebra de la cooperativa o se acuerda el incremento de aportaciones sociales, sin perjuicio de lo dispuesto en el artículo 54».

Posteriormente, Ley 14/2011, de 23 de diciembre, de Sociedades Cooperativas Andaluzas (LCAND), en su artículo 69.2, también prevé de forma expresa que las pérdidas se imputarán al socio hasta el límite de sus aportaciones al capital social.

En la LCCANT, para abordar esta cuestión, se ha partido del concepto moderno de cooperativa como sociedad abierta que puede operar tanto con socios como, en su caso respetando los límites legales, 
con terceros. En efecto, en la actualidad, las cooperativas deber ser conceptuadas como verdaderas sociedades y, por tanto, personas jurídicas, en el sentido de entes superiores y distintos a los sujetos que las integran, dotados del atributo de la personalidad, que les otorga capacidad para obligarse, para actuar, en definitiva, en el tráfico jurídico, siéndoles por ello imputables todos los resultados prósperos o adversos que resulten de su actuación. De forma acorde con ello, de las eventuales pérdidas debe responder todo el patrimonio de la cooperativa, incluidas las aportaciones de los socios. Asimismo, la naturaleza societaria de la cooperativa impide considerar que la pérdida no es de la sociedad, sino del socio directamente. A nuestro juicio, no cabe otra interpretación que la naturaleza societaria de la pérdida; pérdida societaria, sujeta a un particular régimen legal de imputación, que debe incardinarse dentro de las medidas previstas, en este tipo societario, para la protección de los terceros y que debe ser compatible con el régimen de responsabilidad limitada que suelen prever las leyes de cooperativas modernas.

Por tanto, la LCCANT se regula:

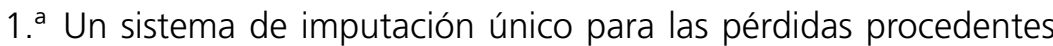
de la actividad económica realizada por la cooperativa con los socios y para resto de las pérdidas societarias.

2. ${ }^{a}$ Una previsión expresa de que las pérdidas se podrán imputar al socio hasta el límite de sus aportaciones al capital social.

Concretamente el artículo 72 de la LCCANT, prevé lo siguiente:

«1. Los estatutos sociales deberán fijar los criterios para la compensación de las pérdidas, siendo válido imputarlas a una cuenta especial para su amortización con cargo a futuros resultados positivos, dentro del plazo máximo de siete años.

2. En la compensación de pérdidas la sociedad cooperativa habrá de sujetarse a las siguientes reglas:

a) A los fondos de reserva voluntarios, si existiesen, podrá imputarse la totalidad de las pérdidas.

b) Si se ha optado por la contabilización separada de los resultados extracooperativos: al fondo de reserva obligatorio podrán imputarse, como máximo, dependiendo del origen de las pérdidas, los porcentajes medios de los excedentes cooperativos o beneficios extracooperativos y extraordinarios que se hayan destinado a dicho fondo en los últimos cinco años o desde su constitución, si ésta no fuera anterior a dichos cinco años.

Si, por el contrario, se ha previsto la contabilización conjunta de los resultados de la cooperativa: al fondo de reserva obli- 
gatorio podrá imputarse, como máximo, el porcentaje medio de los resultados que se hayan destinado a dicho fondo en los últimos cinco años o desde su constitución, si ésta no fuese anterior a dichos cinco años.

c) La cuantía no compensada con los fondos obligatorios y voluntarios se imputará a los socios en proporción a las operaciones, servicios o actividades realizadas por cada uno de ellos con la sociedad cooperativa. Si estas operaciones o servicios realizados fueran inferiores a los que como mínimo está obligado a realizar el socio conforme a lo establecido en el artículo 20.2.b) de esta Ley, la imputación de las referidas pérdidas se efectuará en proporción a la actividad cooperativizada mínima obligatoria.

Las pérdidas se imputarán al socio hasta el límite de sus aportaciones al capital social.

3. Las pérdidas imputadas a cada socio se satisfarán de alguna de las formas siguientes:

a) El socio podrá optar entre su abono directo o mediante deducciones en sus aportaciones al capital social o, en su caso, en cualquier inversión financiera del socio en la sociedad cooperativa que permita esta imputación, dentro del ejercicio siguiente a aquel en que se hubiera producido.

b) Con cargo a los retornos que puedan corresponder al socio en los siete años siguientes, si así lo acuerda la asamblea general. Si quedasen pérdidas sin compensar, transcurrido dicho periodo, éstas deberán ser satisfechas por el socio en el plazo máximo de un mes a partir del requerimiento expreso formulado por el consejo rector».

Con este régimen legal y con la exigencia, prevista en todas las leyes de nuestro país, de mantener la cuantía de la aportación obligatoria para poder seguir siendo socio (por todas, artículo 46 LCOOP o 58.1 LCPV), los afectados, es decir, aquéllos cuya aportación obligatoria, como consecuencia de las deducciones en el capital social para compensar pérdidas, haya quedado por debajo de la cifra fijada por los estatutos o por la asamblea general, deberán realizar el desembolso necesario hasta alcanzar su importe, si quieren mantener su condición._De ese modo, se garantiza la compatibilidad real de la responsabilidad limitada, que suelen prever las leyes de cooperativas modernas, y del sistema de imputación de pérdidas. Con el régimen apuntado, los socios responden únicamente hasta el límite de las aportaciones al capital social que hayan suscrito. La necesidad de realizar aportaciones suplementarias para mantener la cuantía de la 
aportación obligatoria, reducida por pérdidas, sólo es necesaria para mantener su condición dado que para mantener el privilegio de la responsabilidad limitada, los socios deben aportar a la sociedad la cifra de capital adecuada.

\section{El Fondo de Reserva Obligatorio (FRO)}

Como es sabido, el FRO tiene gran importancia en una sociedad de capital variable como la cooperativa, ya que por su estabilidad favorece la conservación y el desarrollo de las actividades empresariales de la sociedad. Además, no puede olvidarse su finalidad de garantía, puesto que con la reserva obligatoria deberán compensarse las pérdidas si no existen otras reservas disponibles. En general, al margen de opiniones interesadas, es comúnmente aceptada la postura seguida por la LCCANT que considera que el FRO, salvo contadas excepciones, debe ser irrepartible, tanto en caso liquidación como de baja. La irrepartibilidad del fondo de reserva obligatorio entre los socios se explica, además, por los objetivos solidarios y de marcada proyección social que persiguen las sociedades cooperativas. Sin embargo, atendiendo las reivindicaciones del sector y apartándose de la Ley General, la LCCANT admite, a nuestro juicio de forma desafortunada, la posibilidad de reducir sus dotaciones a la mitad cuando el fondo de reserva obligatorio supere el 100 por cien del capital social, si lo prevén los estatutos.

\section{Clases de cooperativas}

En lo que respecta a las cooperativas de primer grado, la Ley se aparta del criterio de clasificación de la Ley General como mero catálogo y, con ánimo clarificador, diferencia entre cooperativas de trabajadores, de consumo, de servicios y especiales. Los criterios que determinan la inclusión de cada sociedad cooperativa en uno de los tres primeros tipos mencionados responderán a la cualidad de las personas socias o a la actividad que éstas desarrollen en la empresa, pero, en ningún caso, a la finalidad u objeto social de la cooperativa. El cuarto recoge una categoría denominada cooperativas especiales. En este caso, la propia ley se refiere a modalidades de cooperativas en lugar de a tipos, lo que obedece a que las contempladas en este grupo no son realmente nuevas clases de cooperativas, sino cooperativas del tipo que sea, que reúnen unas condiciones especiales definidas en la propia ley 
para obtener tal consideración. Esta categoría comprende las Cooperativas de iniciativa social y Cooperativas de integración social, las Cooperativas integrales, las Cooperativas mixtas y las Cooperativas de impulso empresarial.

Asimismo, se establece que, además de las contempladas en el título II de la Ley, podrán regularse reglamentariamente otros subtipos, siempre y cuando reúnan unas características singulares que aconsejen la aplicación de un régimen jurídico específico.

Respecto de las cooperativas de trabajo, además de admitir que se constituyan con solo dos socios trabajadores, se flexibiliza el porcentaje de contratación de trabajadores por cuenta ajena, tanto con carácter indefinido como temporal («el número de horas/año realizadas por trabajadores con contrato de trabajo por cuenta ajena de carácter indefinido o temporal no podrá ser superior, en su conjunto, al 60 por ciento del total de las realizadas por los socios trabajadores, teniendo en cuenta que el número de horas/año a realizar por trabajadores con contrato de trabajo indefinido no podrá ser superior al 30 por ciento»: artículo 100.6), al tiempo que se permite superar el número de horas por año realizadas por trabajadores con contrato de trabajo por cuenta ajena por necesidades objetivas de la empresa y por un periodo máximo de tres meses, siempre que se obtenga la autorización de la autoridad competente en materia de cooperativas de la Comunidad Autónoma de Cantabria. El propósito es permitir a las cooperativas responder a las necesidades del mercado y favorecer la contratación de trabajadores.

En el tratamiento de las cooperativas de explotación comunitaria se han tenido en cuenta las recientes innovaciones aportadas por otras normas legales, con el objeto de clarificar en lo posible la inevitable complejidad estructural de esta fórmula societaria.

Respecto a las cooperativas de enseñanza, se admite que puedan asumir la posición de socios colaboradores, entre otros interesados, los alumnos, sus padres o sus representantes legales, así como los exalumnos.

La regulación de las cooperativas de vivienda tiene como principal objetivo impulsar el cumplimiento del derecho constitucional a una vivienda digna, extremando las cautelas que impidan los abusos producidos en ocasiones a través de esta clase de cooperativa.

De la regulación de las cooperativas agrarias cabe destacar las normas sobre las actividades cooperativizadas, permitiendo que los estatutos exijan una participación mínima o exclusiva y un tiempo mínimo de permanencia de los socios en la cooperativa. Las demás cooperativas de servicios, en sus diferentes modalidades, se regulan de una 
forma flexible para dar cabida a proyectos no sólo de tipo industrial o de servicios, sino también a aquellos promovidos por profesionales o artistas.

Las cooperativas de iniciativa social, concebidas como entidades sin ánimo de lucro, se constituyen para la prestación de todo tipo de servicios de naturaleza social, y las de integración social, carentes igualmente de ánimo de lucro, son las formadas mayoritariamente por personas con discapacidad física, psíquica, sensorial o cualquier otro colectivo con dificultades de integración social, con la finalidad de promover la integración social de sus socios.

La regulación de las cooperativas mixtas se introduce para ofrecer soluciones al problema de la participación de una sociedad de capital en una sociedad cooperativa.

Las cooperativas de impulso empresarial pretenden facilitar la innovación social desde un estricto cumplimiento de los principios cooperativos. Se trata de una eficaz herramienta de fomento del emprendimiento de sus socios, capaz de conseguir el afloramiento de servicios que de otro modo permanecerían en el ámbito de la economía informal.

\section{El Consejo Cántabro de la Economía Social}

En consonancia con lo previsto en la Ley 5/2011, de 29 de marzo, de Economía Social, se prevé la creación del Consejo Cántabro de la Economía Social, con funciones de carácter consultivo y asesor para las actividades relacionadas con este ámbito en el territorio de la Comunidad Autónoma. Asimismo, se asigna al propio Consejo conciliar y ejercer el arbitraje en las cuestiones litigiosas que se planteen entre cooperativas, entre éstas y sus socios, o en el seno de las mismas entre sus socios, cuando ambas partes lo soliciten o bien estén obligadas a tenor de lo establecido en sus estatutos sociales.

\section{Reflexión final}

En el momento actual, siguiendo las pautas anteriores, se considera que es posible ofrecer una regulación respetuosa con el tipo o modelo de verdadera sociedad cooperativa y con los instrumentos necesarios, no sólo para que las cooperativas puedan superar una etapa de crisis sino para que la organización cooperativista pueda ocupar un papel relevante dentro de la actividad económica cántabra. Sin embargo, tam- 
bién se ha puesto de manifiesto que aprobar una legislación adecuada es sólo el primer paso; una vez aprobada empieza el verdadero reto. Entonces, el Gobierno regional, una vez reordenadas y delimitadas adecuadamente las competencias relativas al ámbito de la Economía Social, deberá dotarse de una estructura administrativa capaz de ofrecer servicios y soluciones al mundo cooperativo y apostar, de forma decidida, por la divulgación del ideario cooperativo y por la promoción de las sociedades cooperativas como fórmula ventajosa respecto a otras formas jurídicas, favorecedora del autoempleo. 